\title{
Olanzapine-induced Rabbit Syndrome: A Case Report
}

\author{
Parijat Roy ${ }^{1}$, Sagar Karia ${ }^{2}$, Avinash Desousa ${ }^{3}$, Nilesh Shah ${ }^{4}$
}

\begin{abstract}
Rabbit syndrome is an antipsychotic-induced movement disorder associated with long-term exposure of typical antipsychotics and rarely atypical psychotics. We report the case of a 56-year-old female patient who was suffering from a bipolar mood disorder and was well maintained on olanzapine $10 \mathrm{mg}$ per day. She developed rabbit syndrome 1 year after the initiation of the drug.

Keywords: Antipsychotics, Olanzapine, Rabbit syndrome.

Indian Journal of Private Psychiatry (2020): 10.5005/jp-journals-10067-0048
\end{abstract}

\section{INTRODUCTION}

Rabbit syndrome is an antipsychotic-induced movement disorder characterized by the rhythmic movement of the lips resembling the chewing movement of rabbits. ${ }^{1}$ The movements are typically in the vertical plane 4 to $6 \mathrm{~Hz}$ in frequency without involving the tongue. ${ }^{2}$ This movement disorder typically occurs after prolonged exposure to antipsychotics, mostly the typical antipsychotics, with a prevalence rate of 2.3 to $4.4 \%$ and in rare cases atypical antipsychotics. ${ }^{3}$ There have been anecdotal case reports with risperidone ${ }^{4}$, paliperidone $e^{5}$, and citalopram ${ }^{6}$ having been implicated in the past. This condition is often misdiagnosed as tardive dyskinesia, which, however, involves the tongue and is worsened with anticholinergic exposure. ${ }^{7}$ The management of rabbit syndrome includes the reduction of the offending drug and the addition of an anticholinergic agent. The etiology lies in the basal ganglia, particularly substantia nigra pars reticulata, although the exact mechanism is yet to be understood. ${ }^{8}$ We present herewith the case of a 56-year-old female patient with a bipolar mood disorder that developed rabbit syndrome after being stable for 1 year on olanzapine.

\section{Case Report}

A 56-year-old Marathi-speaking married Hindu female, who was educated till 9th standard, homemaker by profession, presented to our outpatient department with of involuntary perioral movements, tremulousness of hands, and slurring of speech for the past 10 days. The patient was being followed up in our outpatient department for a year when she had presented with complaints of decreased need for sleep, overtalkativeness, overfamiliarity, hyper-religiosity, easy distractibility, big talk, and easy irritability for about 15 days before presentation. That episode was the first such episode ever and was not preceded by any depressive episodes. The patient was provisionally diagnosed as having an acute manic episode and admitted to the hospital. Young's Mania Rating Scale (YMRS) was applied, which revealed a score of 38, and she was started on sodium valproate $600 \mathrm{mg} /$ day, olanzapine $10 \mathrm{mg}$ at night, and clonazepam $1 \mathrm{mg} /$ day in divided doses. All routine investigations were done and were within normal limits. Magnetic resonance imaging (MRI) study of the brain did not reveal any abnormalities, and the patient was continued on the same medications with a gradual titration of the doses of sodium valproate to $800 \mathrm{mg} /$ day and olanzapine to

\footnotetext{
${ }^{1-4}$ Department of Psychiatry, Lokmanya Tilak Municipal Medical College, Mumbai, Maharashtra, India
}

Corresponding Author: Avinash Desousa, Department of Psychiatry, Lokmanya Tilak Municipal Medical College, Mumbai, Maharashtra, India, Phone: +91 22 26460002, e-mail: avinashdes888@gmail.com

How to cite this article: Roy P, Karia S, Desousa A, et al. Olanzapineinduced Rabbit Syndrome: A Case Report. Ind J Priv Psychiatry 2020;14(1):43-44.

Source of support: Nil

Conflict of interest: None

$15 \mathrm{mg} /$ day in divided doses each. The patient was discharged after 18 days, with a $100 \%$ recovery and a YMRS score of 10 on discharge. The patient would follow up regularly every 15 to 30 days and was maintained on olanzapine $10 \mathrm{mg}$ and sodium valproate $400 \mathrm{mg}$. Around 10 days before the current visit, the patient started noticing that she would have involuntary rhythmic movements around her lips. The movement would persist throughout the day increasing during work, which involved sustained attention/concentration and would disappear during sleep. She would further have slurred speech and tremulousness of hands, which were never present previously. Due to these symptoms, she would be distressed and have difficulty doing her daily chores, following which she visited us.

On clinical examination, she had bilateral postural tremors, rigidity at the wrist joint (cogwheel type), slurred speech, and fine rhythmic oscillatory movement of the lips without any tongue movement. The perioral movement would exacerbate when she was asked to do some mental calculation.

Her mental status examination did not reveal any psychopathology except distress for the movements. A provisional diagnosis of olanzapine-induced extrapyramidal symptoms with rabbit syndrome was made, and all routine investigations and serum valproate were advised. Olanzapine was stopped, and the patient was asked to follow up 5 days later. In the next follow-up, all routine investigations were within normal limits and serum valproate was $69 \mu \mathrm{g} / \mathrm{mL}$ and the patient had shown a decrease in her symptoms. The patient perceived around $50 \%$ improvements, and on examination there was no rigidity, postural tremors had come down, and there was an $80 \%$ decrease in perioral tremors. She was started on promethazine $25 \mathrm{mg}$ BD in view of the residual symptoms, and the patient was asked to follow up 15 days later. In the 
subsequent follow-up, she had no postural tremors and mild perioral tremulousness persisted. Subsequently, tetrabenazine $25 \mathrm{mg}$ BD was added, and she was maintained on the same dosage. Within a month, the perioral tremors disappeared. Promethazine and tetrabenazine were gradually withdrawn, and the patient was maintained on sodium valproate $400 \mathrm{mg}$ without a relapse of symptoms.

\section{Discussion}

This case report describes a case of rabbit syndrome, and though commonly associated with typical antipsychotics, it may also be caused due to atypical antipsychotics, olanzapine being the drug that has been implicated in this case. It is important for clinicians to realize that, though rare, this phenomenon may occur with atypical antipsychotics that have low extrapyramidal reaction potential. ${ }^{9}$ One needs to be vigilant about the same. It is also interesting that the condition occurred with just $10 \mathrm{mg}$ of olanzapine and after the patient was maintained on the same for 1 year. There was no formal scale used in the assessment of the condition. The efficacy of tetrabenazine in effectively eliminating the residual symptoms persisting even after anticholinergic therapy is another highlight of this case. There have been reports of tetrabenazine being useful in antipsychotic-induced extrapyramidal reactions. ${ }^{10}$ Busy clinicians should be aware of this potential side effect of olanzapine and not delay the treatment once the symptoms are detected.

\section{ACKNOWLedgments}

Nil

\section{References}

1. Schwartz M, Hocherman S. Antipsychotic-induced rabbit syndrome: epidemiology, management and pathophysiology. CNS Drugs 2004;18(4):213-220. DOI: 10.2165/00023210-200418040-00002.

2. Catena M, Fagiolini $A$, Consoli $G$, et al. The rabbit syndrome: state of the art. Curr Clin Pharmacol 2007;2(3):212-216. DOI: 10.2174/ 157488407781668820

3. Dell'Osso MC, Fagiolini A, Ducci F, et al. Newer antipsychotics and the rabbit syndrome. Clin Pract Epidemiol Ment Health 2007;3(1):6. DOI: 10.1186/1745-0179-3-6.

4. Levin T, Heresco-Levy U. Risperidone-induced rabbit syndrome: an unusual movement disorder caused by an atypical antipsychotic. Eur Neuropsychopharmacol 1999;9(1-2):137-139. DOI: 10.1016/s0924977x(98)00016-9.

5. Teng PR, Lai TJ. Paliperidone-related rabbit syndrome. J Clin Psychopharmacol 2011;31(3):379-380. DOI: 10.1097/JCP. ob013e318219263d.

6. Parvin MM, Swartz CM. Dystonic rabbit syndrome from citalopram. Clin Neuropharmacol 2005;28(6):289-291. DOI: 10.1097/01.wnf. 0000191348.10375.41.

7. Altindag A, Yanik M. A case of rabbit syndrome treated with quetiapine. Eur Psychiatry 2005;20(8):574-575. DOI: 10.1016/j. eurpsy.2004.11.014.

8. Wada Y, Yamaguchi N. The rabbit syndrome and antiparkinsonian medication in schizophrenic patients. Neuropsychobiology 1992;25(3):149-152. DOI: DOI: 10.1159/000118825.

9. Lindsey $\mathrm{PL}$, Mehalic J. Psychotropic medication-induced rabbit syndrome. J Psychosoc Nurs Ment Health Serv 2010;48(2):31-36. DOI: 10.3928/02793695-20100201-02.

10. Savitt D, Jankovic J. Tardive syndromes. J Neurol Sci 2018;389:35-42. DOI: 10.1016/j.jns.2018.02.005. 